Meta

Journal des traducteurs

Translators' Journal

\title{
Rapport du comité de terminologie française de l'Ordre des Comptables Agréés du Québec sur l'expression "PresentFairly »
}

Volume 22, numéro 3, septembre 1977

URI : https://id.erudit.org/iderudit/004464ar

DOI : https://doi.org/10.7202/004464ar

Aller au sommaire du numéro

Éditeur(s)

Les Presses de l'Université de Montréal

ISSN

0026-0452 (imprimé)

1492-1421 (numérique)

Découvrir la revue

Citer cet article

(1977). Rapport du comité de terminologie française de l'Ordre des Comptables Agréés du Québec sur l'expression " PresentFairly ». Meta, 22(3), 195-211.

https://doi.org/10.7202/004464ar d'utilisation que vous pouvez consulter en ligne. 


\title{
DROBLIEMES ET SOLUTIONS
}

\author{
RAPPORT DU COMITÉ DE TERMINOLOGIE FRANÇAISE \\ DE LORDRE DES COMPTABLES AGRÉES DU QUÉBEC \\ SUR L'EXPRESSION « PRESENT FAIRLY »*
}

\begin{abstract}
*... Beaucoup de la rigueur apparente de la comptabilité d'entreprise se dissout dans la relativité. Et comment ne pas rendre à celle-ci son dû quand on constate combien les chiffres d'un bilan s'adaptent inévitablement aux buts divers que l'on poursuit, quand on le fait, ou quand on le demande?

René Savatier, le Droit comptable au service de l'homme, p. 314.
\end{abstract}

\section{BRÈVE ANALYSE DE LA PORTÉE DE L'EXPRESSION}

L'expression PRESENT FAIRLY touche à l'essence même de l'opinion $\mathrm{du}$ vérificateur ${ }^{1,2}$. Or, la traduction française de cette expression se bute, au départ, sur l'imprécision même du sens qu'on lui reconnaît au Canada anglais. Celui-ci est tributaire, au reste, de l'usage américain. C'est pourquoi il apparaît

* Le président de l'Ordre des comptables agréés du Québec a donné au Comité de terminologie le mandat d'étudier l'expression present fairly et d'indiquer s'il y avait lieu de remplacer l'expression française en usage, s présentent fidèlement \$, par une autre. Pour satisfaire les exigences de publication, le rapport du comité est publié sans ses annexes et quelques notes explicatives ont été ajoutés.

1. Mary E. Murphy, Auditing and Theory, p. 192 et Howard F. Stettler, Auditing Principles, Third Edition, p. 14.

2. L'expression à l'étude est tirée du paragraphe d'énoncé d'opinion du rapport type du vérificateur :

In my opinion, these financial statements present fairly the financial position of the company as at..., $19 \ldots$ and the results of its operations and the changes in its financial position for the year then ended in accordance with generally accepted accounting principles applied on a basis consistent with that of the preceding year.

A mon avis, ces états financiers présentent fidèlement la situation financière de la companie au..., 19.. ainsi que les résultats de son exploitation et l'évolution de sa situation financière pour l'exercice terminé à cette date selon les principes comptables généralement reconnus, appliqués de la même manière qu'au cours de l'exercice précédent.

(Manuel de l'I.C.C.A., chapitre 5400.) 
difficile de ne pas considérer le point de vue américain même si, au plan strictement juridique, pareille démarche pourrait être contestable. La politique hésitante de l'I.C.C.A. $^{3}$ relativement à l'acceptation suivie du rejet de la notion de double opinion a récemment montré, s'il en était besoin, l'intérêt de tenir compte d'abord des tendances d'outre-frontières. Il conviendra, ensuite, de jeter un coup d'œil sur la pratique française et britannique et de s'arrêter, enfin, aux usages du milieu canadien.

\section{1. ÉTATS-UNIS}

Même si les principales étapes de l'évolution observée aux États-Unis depuis le début du siècle ne manquent pas d'intérêt ${ }^{5}$, il suffira ici de s'arrêter aux précisions apportées en 1970 dans le Statement No. $4^{\circ}$. Selon cette prise de position, les états financiers répondront aux exigences de l'expression PRESENT FAIRLY IN CONFORMITY WITH GENERALLY ACCEPTED ACCOUNTING PRINCIPLES lorsque :

$1^{\circ}$ l'on aura respecté les principes comptables généralement reconnus dans la manière de recueillir et de traiter l'information comptable, compte tenu des circonstances dans lesquelles se trouve l'entreprise en cause ;

$2^{\circ}$ l'on aura indiqué convenablement les changements apportés aux principes comptables généralement reconnus durant l'exercice en cause;

$3^{\circ}$ les états financiers reflèteront et présenteront l'information contenue dans les registres comptables d'une manière :

a) qui traduise bien ce qu'on trouve dans de tels registres;

b) qui réponde aux exigences des principes comptables généralement reconnus;

$4^{\circ}$ l'on aura répondu dans un juste rapport aux deux exigences opposées, qui sont les suivantes :

a) la nécessité de faire connaître, d'une manière conforme aux principes comptables, les aspects importants, relatifs à la situation financière et aux résultats de l'exercice;

b) la nécessité de communiquer d'une manière synthétique et en la forme coutumière (les états financiers) de grandes masses de données.

Il se dégage de ce texte :

$1^{\circ}$ une idée de conformité. Les états financiers, dit-on, doivent être conformes :

a) aux principes comptables généralement reconnus (c'est la norme) ;

3. Institut canadien des comptables agréés.

4. Par double opinion, on entend que le vérificateur émet une opinion sur la fidélité de la présentation et une autre sur la conformité aux principes comptables généralement reconnus. L'I.C.C.A. a rejeté cette notion et considère que le vérificateur ne doit exprimer qu'une seule opinion, la fidélité et la conformité formant un tout indissociable.

5. Voir Annexe A dans le rapport complet.

6. Publication de l'American Institute of Certified Public Accountants (AICPA). 
b) à la réalité, c'est-à-dire aux faits notés dans les registres ;

c) à l'objectif de permanence dans l'application des principes comptables retenus d'un exercice à l'autre, à défaut de quoi toute divergence devra être signalée ;

$2^{\circ}$ une idée de choix dans l'information transmise qui ne doit pas appauvrir celle-ci au point de masquer des faits importants qui, par leur nature ou leur valeur, doivent être communiqués au lecteur des états financiers.

Il se dégage, en outre, du texte en cause que l'expression est utilisée dans une périphrase qui forme un tout. L'AICPA $^{7}$ consacre ainsi une interprétation que l'on trouvait déjà chez des auteurs comme Stettler et Murphy.

Pour Stettler ${ }^{8}$, l'expression PRESENT FAIRLY IN CONFORMITY WITH GENERALLY ACCEPTED ACCOUNTING PRINCIPLES signifie, d'une manière générale, que les états financiers :

a) doivent refléter une connaissance des faits (factual). On retrouve donc l'idée de conformité à la réalité ;

b) doivent faire connaître tous les renseignements essentiels (disclose all essential information). On retrouve ici à la fois l'idée de conformité à la réalité et l'idée de choix;

c) ne doivent pas induire en erreur, ni tromper le lecteur moyen (misleading to the average reader), ce qui met en cause l'idée de choix.

De son côté, Mary Murphy avance que l'expression PRESENT FAIRLY ${ }^{9}$ veut dire que l'image globale des états financiers reflète d'une manière acceptable, quant aux principes comptables, la situation sans prétendre à l' « exactitude » (exactness). Cette position rejoint le jugement d'un tribunal anglais ${ }^{10}$ dans lequel on a indiqué que le vérificateur a la responsabilité de dire si le bilan est à la fois mathematically accurate et substantially accurate. On rejoint de nouveau l'idée de conformité.

Des textes qui précèdent, on voit nettement qu'une "marge d'interprétation » est laissée au jugement de l'expert-comptable. Ce sera l'essence même de son opinion que de se prononcer en définitive sur le tri, le classement et le regroupement des données sous la triple fidélité aux faits, aux principes comptables et à la permanence dans l'application de ceux-ci.

\section{ROYAUME-UNI}

On a l'habitude de considérer le Royaume-Uni comme le berceau du public accountancy moderne. En effet, dès le milieu du siècle dernier, commence à se constituer dans le domaine comptable une profession organisée. Le fait que ce pays ait servi de modèle à plusieurs autres constitue à lui seul une

7. American Institute of Certified Public Accountants.

8. Cf. op. cit., p. 14 .

9. Cf. op. cit., p. 192 et 51.

10. Cause Leeds Estate Building and Investment Co. V. Shepherd (36 Ch. 787), citée par Mary Murphy, op. cit., p. 51 . 
raison suffisante pour qu'on s'y attarde. À ce fait s'ajoute, du moins en ce qui concerne le Canada, l'indéniable influence qu'ont exercée les organisations d'accountants britanniques sur les membres de la profession.

En 1900, la loi britannique vient consacrer une pratique largement répandue en obligeant les compagnies à soumettre leur bilan au contrôle d'un accountant. Toutefois, ce n'est que beaucoup plus tard, avec la mise en vigueur de directives particulières citées dans la Companies Act de 1948, que le législateur statuera sur les exigences auxquelles doit alors satisfaire l'auditor. Ce document qui, à maints égards, marque le début d'une ère nouvelle renforce singulièrement la sévérité de l'audit aussi bien que les exigences vis-à-vis de la capacité professionnelle de l'auditor.

À l'intérieur du cadre général auquel nous venons brièvement de nous référer, qu'en est-il en matière de rapport du vérificateur? Jusqu'en 1929 , il est fréquent de voir l'accountant approuver un bilan en y faisant paraître sa signature précédée des mots audited ou certified. Lors des changements apportés à la Companies $A c t$ en 1929, on a indiqué les différents points sur lesquels doit porter le rapport du vérificateur. L'Institute of Chartered Accountants in England and Wales, s'inspirant de cette disposition de la loi, propose un texte qu'on utilisera jusqu'au moment de la révision de 1948 de la Companies Act. On lui a alors substitué la formule suivante :

In our opinion and to the best of our information and according to the explanations given to us, the annexed Balance Sheet and Profit and Loss Account give a true and fair view of the state of the Company's affairs as at 31 st December, $19 \ldots$, and of its profit for the year ended on that date. We have obtained all the informations and explanations which to the best of our knowledge and belief were necessary for our audit. In our opinion, the Company has kept proper books of account, and the above-mentioned accounts, which are in agreement therewith, give in the prescribed manner the information required by the Companies Act, $1948^{11}$.

On observe d'abord l'emploi des mots true and fair view. Ensuite, on remarque sans aucun doute qu'on ne fait aucune différence aux principes comptables généralement reconnus.

D'autres changements apportés, cette fois en 1967, à la Companies Act ne modifieront pas cette situation, pas plus que la position que prendra le 7 août 1968 l'Institute of Chartered Accountants in England and Wales. Cet organisme donnera en effet dans Auditor's reports : forms and qualifications l'exemple d'un rapport sans réserve :

In our opinion, the accounts set out on pages... to... together give, so far as concerns members of the holding company, a true and fair view of the state of affairs at... and of the profit (or loss) for the year ended on that date and comply with the Companies Act 1948 and $1967^{12}$.

L'expression true and fair view est donc présentement utilisée. «The auditor is required to report whether in his opinion the accounts give a true and fair

11. Cité par Antoine Mas, Introduction da la révision des entreprises, p. 180.

12. The General Educational Trust of the Institute of Chartered Accountants in England and Wales, Survey of Published Accounts, 1971-1972, p. 160. 
view. There is no definition of true and fair view. Compliance with generally accepted accounting principles is implied ${ }^{13}$.

\section{FRANCE}

En France, on s'appuie sur une législation qui fait expressément mention des deux qualités exigées des comptes sociaux : la régularité et la sincérité. La loi $\mathrm{n}^{\circ} 537$ du 24 juillet 1966 fait appel à ces deux notions pour préciser l'objet de la certification des commissaires aux comptes quand, à l'article 228 , elle indique ce qui suit ${ }^{14}$ :

Alinéa $1^{\text {er }}$ : Les commissaires aux comptes certifient la régularité et la sincérité de l'inventaire, du compte d'exploitation générale, du compte de pertes et profits et du bilan.

Alinéa 2: Ils ont pour mission permanente, à l'exclusion de toute immixtion dans la gestion, de vérifier les livres et les valeurs de la société et de contrôler la régularité et la sincérité des comptes sociaux.

Dans leur rapport présenté en 1971 au congrès de l'Ordre des conseils fiscaux de France, MM. Puyraveau et Descottes-Genon retiennent, avec pertinence, la distinction entre exactitude, régularité et sincérité :

On n'a pas voulu, disent-ils, imposer aux sociétés l'obligation de présenter un bilan exact - l'exactitude étant une notion bien trop absolue dans un domaine où l'approximation est souvent inévitable - pour n'exiger que la régularité et la sincérité, et c'est déjà beaucoup ${ }^{15}$.

\section{Régularité}

Ils rappellent, ensuite, que "la régularité c'est la conformité à la règle du droit » (Dictionnaire Larousse). Le Dictionnaire Robert ${ }^{16}$ donne, notamment, ce qui suit : "caractère, qualité d'une chose ou d'une personne qui est régulière ; conformité aux règles. Régularité d'une élection ». MM. Puyraveau et DescottesGenon ajoutent que «la régularité s'appréciera eu égard aux règles fixées par la loi, la jurisprudence, le Conseil national de la comptabilité et les organisations professionnelles compétentes pour préciser le contenu de la doctrine comptable *. Ils devancent les propos de M. Goré qui écrit, deux ans plus tard :

D'une façon générale la régularité doit être appréciée essentiellement au regard des règles du droit commercial...

La régularité doit être appréciée au regard non seulement des dispositions légales ou réglementaires, mais de la pratique habituelle. Celle-ci est exposée dans les ouvrages et les publications périodiques. Elle est fondée sur

13. Accountants International Study Group, International Financial Reporting, paragraphe no 19.

14. Cité par François Goré, « Les notions de régularité et de sincérité des comptes », dans Revue française de comptabilité, no 25 , avril 1973 , p. 168.

15. M. Puyraveau et $\mathrm{M}$. Descottes-Genon, Incidences du droit fiscal sur la régularité et la sincérité des bilans, Rapport présenté au congrès de l'Ordre des conseils fiscaux đe France, le 13 mai 1971.

16. Le Robert, tome IV. 
l'expérience des praticiens et l'évolution des divers facteurs qui déterminent leurs décisions (évolution des sciences, des techniques, du droit).

Ici, il convient de souligner la valeur toute particulière qui s'attache aux notes d'information du Conseil national de la comptabilité, aux recommandations à l'usage des membres de l'Ordre des experts comptables, adoptées par le Conseil supérieur de l'Ordre des experts comptables et des comptables agréés; la pratique habituelle influence les solutions qui sont suggérées, et celles-ci influencent de leur côté les praticiens dans l'exercice de leur profession. Ces organes précisent progressivement le contenu de la doctrine comptable.

Des usages se forment ainsi parmi les professionnels. Ce sont des usages de fait, et non des usages de droit, des coutumes. Ces usages ne deviennent des coutumes que lorsqu'ils sont sanctionnés par la jurisprudence, quand les tribunaux ont à connaître des procès au cours desquels la régularité de comptabilités commerciales doit êtrc appréciée. Ils n'en sont pas moins importants, car ils constituent une référence pour l'appréciation de la régularité des comptes et des documents que les commissaires aux comptes sont appelés à certifier en application de l'article 228 de la loi de 1966.

I1 convient à cet égard de noter un des principes fondamentaux généralement admis, qui est un véritable principe coutumier : le principe de fixité. Les comptes, et en particulier ceux qui enregistrent des évaluations, doivent être comparables dans le temps afin de remplir au maximum leur fonction d'information des actionnaires. Les valeurs figurant au bilan ont, en effet, un caractère relatif : c'est moins leur valeur absolue que leur évolution qui retient le plus souvent l'attention des utilisateurs.

Un des principes fondamentaux à respecter est donc le maintien des mêmes règles d'un exercice à l'autre, ce qui permet d'apprécier l'évolution des résultats et du patrimoine de l'entreprise examinée. Ce principe a d'ailleurs été consacré par l'article 341 de la loi de 1966 sur les sociétés commerciales : article 341 , alinéa $1^{\mathrm{er}}$ 《Le compte d'exploitation générale, le compte de pertes et profits et le bilan sont établis à chaque exercice selon les mêmes formes et les mêmes méthodes d'évaluation que les années précédentes.

Mais cette fixité est relative. L'article 341, alinéa 2, de la loi de 1966 permet de modifier les formes et les méthodes d'évaluation à certaines conditions de formes prévues par ce texte et l'article 244 du décret d'application ; cet article déclare en particulier : «Si d'autres méthodes que celles prévues par les dispositions en vigueur ont été utilisées pour l'évaluation des biens de la société dans l'inventaire et le bilan, il en est fait mention ${ }^{17}$.

Quant à la dérogation possible aux règles habituelles de présentation et de méthodes comptables, l'auteur ajoute que «la comptabilité étant une technique d'information, le critère est le souci d'informer, notamment, dans les sociétés, les associés, les actionnaires. Les tableaux de synthèse présentés seront réguliers tant que la forme et la méthode adoptées ne donneront pas une image infidèle de la situation de la société... La régularité est absorbée ici dans la sincérité ${ }^{18}$.

On aura sans doute remarqué que pour expliquer sa pensée, l'auteur fait appel au mot INFIDĖLE.

17. François Goré, op. cit., p. 169.

18. Cf. op. cit. p. 170. 


\section{Sincérité}

«Quant à la sincérité, disent MM. Puyraveau et Descottes-Genon, dès lors qu'on la distingue de l'exactitude, c'est une notion qui relève des qualités morales et qui suppose un jugement d'appréciation et de valeur. Elle s'apprécie alors beaucoup plus en fonction des responsables des comptes que des comptes euxmêmes ${ }^{19} . \gg M$. Goré reconnaît la difficulté de cerner la notion de sincérité. Purement subjective, elle s'oppose, à cet égard, au mot exactitude auquel «s'attache une rigueur qui exclut toute nuance ${ }^{20} »$. Selon le garde des Sceaux, M. Pleven, « le commissaire atteste que les documents dressés sont sincères, c'està-dire... que pour les comptes par nature approximatifs, il vérifie la bonne foi des dirigeants dans le choix des options et des évaluations... ${ }^{21}$ ». En revanche, la Commission des opérations de bourse s'en tient, dans un rapport publié en 1969, à une appréciation objective.

Face à ces deux théories, M. Goré prend position et affirme que, sur le plan du droit privé, la sincérité doit être appréciée objectivement. I1 ajoute :

La sincérité se situe au niveau du choix des règles, lorsque ces règles ne sont pas obligatoires.

C'est donc un critère objectif qui doit être utilisé sur le plan du droit privé.

À l'étranger, on emploie le terme fidèle, au lieu de sincère ${ }^{22}$.

Il nous a paru important d'accorder une place relativement large à la France afin de fournir un certain nombre d'éléments propres à préciser convenablement le cadre dans lequel s'emploient les mot régularité et sincérité. De ce qui précède, il semble se dégager que la marge laissée au jugement de l'expert comporte des limitations découlant de la vision largement juridique de son rôle.

\section{CANADA}

L'I.C.C.A. a suggéré, pour la première fois, une formule de rapport du vérificateur dans un bulletin paru en 1951 et révisé en 1954. On y emploie la formule suivante : "...exhibit a true and correct view».

Lorsque, quelques années plus tard, nos confrères anglophones ont commencé à utiliser present fairly, ils voulaient par là abandonner l'expression \& exhibit a true and correct view», considérée comme impliquant un degré d'exactitude irréaliste, sans toutefois vouloir diminuer la responsabilité du vérificateur. Il s'agissait en somme d'admettre implicitement que l'opinion de celui-ci se fonde parfois sur des estimations et approximations, tout en reconnaissant qu'il est quand même en mesure de conclure que les états sont exempts d'erreur ou d'omission de quelque importance. C'est ce qu'a reconnu l'I.C.C.A. lorsqu'il a publié en octobre 1959 le Bulletin $\mathrm{n}^{\circ} 17^{23}$. On observe, en outre, l'introduction

19. M. Puyraveau et M. Descottes-Genon, op. cit.

20. François Goré, op. cit., p. 170.

21. M. Pleven, Revue française de comptabilité, octobre 1970, p. 5 et suivantes.

22. François Goré, op. cit., p. 171.

23. Les bulletins de 1'I.C.C.A. étaient publiés par le Comité des recherches en comptabilité et vérification de cet organisme. Ils ont été remplacés par le Manuel de l'I.C.C.A. 
par ce bulletin dans le rapport du vérificateur des mots « in accordance with generally accepted accounting principles applied on a basis consistent with that of the preceding year ». Comment interpréter ce que l'on entendait alors par «accounting principles»? Nous ne croyons faire mieux que de nous en remettre à deux auteurs qui, à ce moment, ont exprimé leurs vues. Dell et Wilson écrivaient, en effet, naguère ce qui suit :

... there is a body of conventions or rules which must be followed and which very definitely limits the freedom of action of the accountant.

The body of conventions or rules used by accountants is referred to as accounting principles. These principles should be followed whenever financial statements are prepared. Because of the importance of ensuring that this criterion has been met, the Committee on Accounting and Auditing Research of the Canadian Institute of Chartered Accountants, in its Bulletin No. 17, recommended that all auditor's reports include, as part of the auditor's opinion, a specific statement as to whether the financial statements have been prepared «in accordance with generally accepted accounting principles».

In many ways it is unfortunate that the accounting profession adopted the use of the word «principles》 to describe the body of procedures within which it operates. It is unfortunate because the word "principles 》 has more than one meaning, and the meaning which is intended in the phrase "accounting principles» or "principles of accounting》 is not the one most commonly in use. In ordinary conversation the word "principle» is most often used in the sense of meaning those unchanged standards of conduct from which no deviation can be permitted. Thus, we speak of «a man of high principles ». But this is not the only sense or meaning of the word, for «principles» also means « a general rule or law as a guide to action 》, and in this sense the word has been used by the legal profession for many years in the phrase "principles of law». It is only in this sense that the phrase «accounting principles》 can be understood.

Many accountants think that it would be better to refer to accounting procedures rather than accounting principles, but it is probably too late now to change our terminology. The phrase has become part of the language of business and law, and we must therefore use it in practice; but in using it we must be stare to understand that it means only what it is intended to mean and nothing more. This might seen an arbitrary dictum, but it is not the only phrase in the English language which has a meaning different from the sum of the meanings of the underlying words ${ }^{24}$.

Parallèlement, en laissant tomber « de manière à représenter l'état véritable et exact », les francophones, dans la version française de ce bulletin $\mathrm{n}^{\circ} 17$, adoptaient présentent équitablement, expression remplacée à son tour, en septembre 1967, dans le bulletin $\mathrm{n}^{\circ} 25$ par présentent fidèlement. Parfois, en certaines occasions, on s'est même servi de présentent objectivement, phénomène accidentel sans doute car aujourd'hui on déroge rarement à présentent fidèlement.

Enfin, l'abolition de la notion de double opinion que propose l'exposésondage de novembre 1975 intitulé Rapport du vérificateur : le rapport type et qui a été intégrée au Manuel en décembre 1976 avec la publication du Cha-

24. H.C. Dell, C.A., J.R.M. Wilson, C.A., Auditing Procedures, Third Edition, p. 21-22. 
pitre 5400 mérite d'être signalée car elle marque, sur ce point, une correspondance avec la position américaine. Il ne s'agit pas de rendre uniquement present fairly, mais bien de rendre en entier le message que nous soulignons dans le texte du paragraphe d'énoncé d'opinion du vérificateur reproduit ci-dessous :

In my opinion, these financial statements present fairly the financial position of the company as at... 19.. and the results of its operations and the changes in its financial position for the year then ended in accordance with generally accepted accounting principles applied on a basis consistent with that of the preceding year.

Pour ceux qui hésiteraient encore, le Comité des normes de vérification de l'I.C.C.A. affirme qu'il ne s'agit pas pour le vérificateur de déclarer d'une part que les états financiers sont sincères, fidèles, etc., et d'autre part qu'ils sont conformes aux principes comptables généralement reconnus. En d'autres termes, "...present fairly... in accordance with generally accepted accounting principles 》 forme un tout. De plus, au paragraphe 12 du chapitre 5400, on lit: "In the auditor's standard report, the auditor's judgement concerning «present fairly» can be applied only within the framework of generally accepted accounting principles.» Il résulte que, dans le tout dont nous venons de parler, l'on se réfère essentiellement aux principes comptables généralement reconnus pour indiquer l'optique dans laquelle se place le vérificateur lorsqu'il déclare que "...les états financiers présentent fidèlement... ». On lève, en conséquence, l'ambiguïté de la double opinion en indiquant que la référence aux principes comptables généralement reconnus ne constitue pas une exigence qui viendrait s'ajouter mais une indication de l'optique dans laquelle le vérificateur se place lorsqu'il déclare que la présentation est conforme à l'objectif qu'exprime le mot fairly. L'I.C.C.A. rejoint ici la prise de position de l'AICPA émise en juillet 1975 dans Statement on Auditing Standards - 5 par l'Auditing Standards Executive Committee.

\section{EXAMEN CRITIQUE}

\section{Observations préalables}

Nous avons examiné le problème de la traduction de PRESENT FAIRLY en orientant notre réflexion sur la responsabilité du vérificateur et sur les exigences d'une pensée française en milieu nord-américain.

A l'égard de la responsabilité du vérificateur, il nous est apparu que la traduction française de l'expression en cause ne devait pas placer l'expert dans une situation différente selon la langue de rédaction du rapport. Il saute aux yeux qu'il serait injuste de donner à la traduction française une clarté plus grande que celle que l'on observe dans le texte de la langue d'origine, l'anglais. Il résulte de ceci que les considérations linguistiques ne sont pas les seules qui sont intervenues dans notre examen de la question. Compte a été tenu, en conséquence, de la charge juridique.

Nous avons également procédé à une étude approfondie du vocabulaire utilisé en France. Nous croyons devoir dire que toute référence à la situation 
impose de reconnaître que là-bas le législateur a promulgué des lois dont les textes contiennent, nous l'avons vu dans la première partie, les mots régularité et sincérité. Ces deux termes s'insèrent dans un cadre juridique. Pareille insertion contribue à leur donner, dans ce milieu, une force que l'on ne retrouve pas chez nous, d'où une invitation au discernement.

Le terme $F A I R L Y$ englobe les notions de vérité, de justice, d'équité, de sincérité, de franchise, d'impartialité. D'après le Webster, il semble que l'on puisse retenir trois sens pertinents :

a) in conformity with the evidence,

b) without fraud,

c) without bias or distortion.

Du point de vue de ceux qui ont la responsabilité de dresser les états financiers sur lesquels portera le jugement du vérificateur, on peut dégager :

a) un élément moral qui relève de l'ordre des intentions et qui touche à l'honnêteté, à l'objectivité ;

b) un élément matériel qui englobe l'information fournie, mettant ainsi en cause le caractère plénier ou partiel des renseignements présentés en tenant compte de leur importance et le respect des méthodes utilisées antérieurement.

Nous avons vu qu'en France, on avance une théorie qui met en valeur l'élément moral avec une clarté que nous n'avons pas encore observée dans notre milieu.

\section{Expressions}

Une dizaine d'expressions envisageables ont retenu notre attention. En voici la liste :
1) justement
2) équitablement
3) loyalement
4) correctement
5) objectivement
6) sincèrement
7) régulièrement
8) fidèlement
9) bien
10) convenablement

Nous nous bornerons à indiquer pourquoi nous n'avons pas retenu la plupart d'entre elles.

\section{Justement}

Dans son Dictionnaire des difficultés grammaticales et lexicologiques, Joseph Hanse rappelle que : 
Justement a un sens spécial : avec justice, avec équité. Châtiment justement infligé. En dehors de ce cas, il peut avoir le même sens que l'adverbe JUSTE, mais l'usage établit des distinctions.

Le Dictionnaire Robert éclaire le texte qui précède en disant : «Avec justice, conformément à la justice : être justement puni ${ }^{25}$. »

L'exemple qui accompagne chacune des deux citations qui précèdent indique que le mot JUSTICE est utilisé en se référant à un «principe moral de conformité au droit positif ${ }^{26} \gg$. L'adverbe JUSTEMENT comporte une nuance morale et de ce fait ne traduit pas entièrement l'idée qu'il faut rendre.

\section{Équitablement}

Comme le terme précédent, le mot EQUITABLEMENT introduit une notion de conformité au droit. Il y a de plus l'idée d'un rapport entre deux êtres. Agir équitablement, c'est rendre à chacun son dû. Or, ce n'est pas ce que l'on cherche à exprimer. Le vérificateur n'a pas à effectuer de partage entre des individus. Il a à émettre une opinion sur l'ensemble d'une situation dont le caractère d'équité ne peut, tout au plus, qu'être un élément parmi plusieurs autres.

\section{Loyalement}

Le Robert indique au mot LOYAL : "Conforme à la loi, ce qui est requis par la loi » et renvoie au mot LEGAL. L'exemple qu'il donne se réfère au droit commercial : "Qualité loyale et marchande. Ce blé n'est pas loyal, il y a trop de seigle... il est plein de charançons. » Cet adverbe transmet la notion de légalité, notion qui ne rejoint pas celle que FAIRLY exprime.

\section{Correctement}

Le Robert donne à l'adverbe CORRECTEMENT ce qui suit : "Sans faute, d'une manière correcte ${ }^{27}$. $\AA$ A l'entrée CORRECT, on trouve notamment :

Qui respecte les règles dans un domaine déterminé. Conforme à l'original.

Qui observe certaines règles arbitrairement définies: dessin correct. Au figuré, qui est conforme aux règles morales, aux usages, aux mœurs.

Le Larousse du $X X^{e}$ siècle ${ }^{28}$ donne en substance ce qui précède mais reconnaît au figuré le sens de «juste, fidèle, exact» au mot CORRECT. Sans doute $\mathrm{M}$. Antoine Mas, professeur à l'Université de Louvain, avait-il à l'esprit cette définition lorsqu'il traduisit le short-form of accountant's report recommandé en 1948 par l'AICPA pour répondre aux exigences de la Securities and Exchange Commission. Dans cette traduction, on lit :

... À notre avis, le bilan et le compte de profits et pertes ci-joints présentent correctement la situation financière de la Société $X$ au 31 décembre 19 .. et le résultat de ses opérations pour l'exercice, clôturé à la même date, con-

25. Le Robert, tome IV, p. 396.

26. Ibid., sous l'entrée " justice *.

27. Cf. op. cit., tome I, p. 964 .

28. Larousse du $X X^{\ominus}$ siècle, vol. 2, p. 492. 
formément à des principes comparables généralement admis appliqués avec continuité pendant les deux derniers exercices ${ }^{29}$.

Nous sommes en face d'un terme dont le sens ne paraît pas arrêté d'une manière aussi claire que les précédents. La définition que le Larousse lui attribue au sens figuré tend à rejoindre l'idée que l'on cherche à exprimer. À l'analyse, on se rend compte que cette situation découle de l'insertion du mot fidèle dans la définition. Qu'on enlève le mot fidèle et les hésitations disparaissent et conduisent au rejet de l'expression. Ce rejet se fonde sur le refus d'associer au rapport du vérificateur la notion

a) de justice, dont nous venons de traiter au mot JUSTEMENT ;

b) d'exactitude, qui comporte une rigueur qui exclut toute nuance, comme l'a indiqué $\mathrm{M}$. Goré ${ }^{30}$.

Mais ce raisonnement ne fait pas disparaître la synonymie entre CORRECTEMENT et FIDĖLEMENT qu'exprime le Larousse. Dans l'opération de première élimination qui caractérise notre démarche, nous ne pouvons pas rejeter CORRECTEMENT. Pour l'instant, elle reste en suspens et sera reprise plus loin.

\section{Objectivement}

$\mathrm{Au}$ sens large, c'est-à-dire "l'absence de parti pris ${ }^{31}$ », le Robert donne : «D'une façon objective, impartiale ${ }^{32}$.» Dans la présentation des comptes d'une entreprise, l'objectivité peut théoriquement coexister avec une marge d'erreur intolérable. Il ne suffit pas que la direction de l'entreprise ait une visée objective en rédigeant les états. Le souci d'objectivité l'empêche sans doute de triturer les données en vue de produire un effet mais il ne prévient pas nécessairement certaines erreurs ou omissions que le vérificateur jugerait importantes. Dans le cas de placements risqués, par exemple, le vérificateur pourrait bien déclarer que les états présentent objectivement la situation, tout en jugeant que la provision pour pertes est insuffisante.

L'objectivité est proche de la notion de choix ${ }^{33}$, sans aucun doute, mais l'idée de conformité y trouve-t-elle son compte? Cela paraît moins sûr. En outre, « on dit, par extension, de quelqu'un qu'il a l'esprit objectif et qu'il sait se placer à un point de vue objectif, lorsqu'il ne se laisse pas entraîner par ses préférences individuelles ${ }^{34}$. Peut-on exclure toute préférence individuelle dans la présentation des comptes? La réponse est, dans une certaine mesure, négative. Dans cette perspective, l'adverbe OBJECTIVEMENT ne nous semble pas devoir être retenu.

\section{Sincèrement - Régulièrement}

SINCËREMENT et SINCÉRITÉ ne peuvent se dissocier. Nous avons pris soin d'insister précédemment sur le fait qu'en France le mot SINCÉRITÉ figure

\footnotetext{
29. Antoine Mas, Introduction à la révision des entreprises, p. 167.

30. Cf. Supra, p. 9.

31. Larousse du $X X^{e}$ siècle, vol. 5, p. 152

32. Le Robert, tome IV, p. 845 .

33. Cf. Supra, p. 4.

34. Larousse du $X X^{e}$ siècle, vol. 5, p. 151
} 
dans des textes de loi alors que rien de tel n'existe chez nous. Nous avons également fait état des deux théories qui, dans ce pays, entourent le mot SINCERITÉ.

À défaut d'un appui juridique semblable dans notre milieu, il faut reconnaître la difficulté d'échapper aux sens courants que l'on attribue à ces mots. Or, à cet égard, on observe une insistance sur les sentiments, ce qui n'est pas pertinent dans les circonstances. On donne, en outre, au mot SINCËRE la définition suivante : "Authentique, non truqué. Créance sincère et véritable. Un rôle de contributions sincère. Elections générales et sincères ${ }^{35}$. 》

Mais sincèrement, employé seul, ne reflète pas nécessairement l'idée de réalité, de bonne mesure, d'exactitude. C'est pourquoi il est souvent accolé au mot régulièrement.

La vérification des comptes obéissant à des techniques bien précises devra aboutir à certifier ou ne pas certifier la régularité et la sincérité des comptes ${ }^{36}$.

Si nous voulons introduire ici comme en France le concept de régularité, nous sommes forcés de faire une incursion dans le second volet de l'expression d'énoncé d'opinion du vérificateur, où il est question des principes comptables généralement reconnus. Une telle incursion sera possible au Canada si l'I.C.C.A. abandonne " the two-part audit opinion ». La section dans laquelle le vérificateur déclare qu'à son avis de tels états présentent fidèlement sera ainsi lue en conjonction avec l'autre volet, comme c'est le cas aux États-Unis.

L'idée de conformité aux principes comptables généralement reconnus serait rendue par le mot régularité, d'autant plus que la nouvelle Loi fédérale sur les corporations commerciales donne au Manuel de l'I.C.C.A. une portée juridique.

Il semble, toutefois, plus difficile dans notre milieu de suivre le raisonnement de MM. Puyraveau et Descottes-Genon ${ }^{37}$ lorsqu'ils citent $M$. le garde des Sceaux qui a précisé que «le commissaire aux comptes atteste que les documents dressés sont sincères, c'est-à-dire que, pour les comptes par nature approximatifs, il vérifie la bonne foi des dirigeants dans le choix des options et évaluations ». La recherche de l'objectivité, exprimée par le milieu nord-américain, ne nous a pas habitués à mettre en cause d'une manière aussi explicite la bonne foi des administrateurs et dirigeants d'entreprises. Cela n'a toutefois pas empêché l'emploi du mot fidèlement qui, nous allons le voir, contient une référence à la bonne foi, à l'honnêteté.

\section{Fidèlement}

Reconnaissons que FIDËLEMENT prend le départ avec une bonne longueur d'avance. Depuis plusieurs années déjà, il a éliminé à peu près tous ses concurrents et l'habitude du succès en a fait le grand favori des experts-comptables canadiens. Mais cette préférence est-elle bien fondée ou appelle-t-elle des réserves?

35. Le Robert, tome VI, p. 448.

36. La Presse de Tunis, 18 septembre 1973.

37. Cf. Supra, p. 9. 
Si l'on s'en remet au Petit Robert, fidèle se dit d'une personne qui ne manque pas à ses engagements, à la foi donnée; une chose est fidèle si elle est conforme à la vérité ou ne s'en éloigne pas; quant à l'adverbe FIDËLEMENT, il a les mêmes sens que l'ajectif dont il dérive. Le Petit Larousse confirme ces deux acceptions.

Or, en y regardant de près, on constate que ces deux idées participent de la notion de fidélité comptable. En effet, la conformité des états financiers à la réalité ne saurait être l'effet du hasard : elle repose nécessairement sur la tenue d'une comptabilité méthodique, destinée à la reddition sincère des comptes dus par les administrateurs à leurs commettants.

On voit donc que la conformité et la sincérité sont indissociables, la seconde demeurant subordonnée en finalité à la première. Pour peu qu'on prenne le soin de préciser la règle que l'on a observée pour la présentation de l'information financière (les principes comptables généralement reconnus), l'adverbe FIDĖLEMENT nous paraît donc parfaitement adéquat à sa mission de traduire la composante matérielle (conformité), la composante formelle (régularité) et la composante morale (sincérité) de la notion complexe cxprimée en anglais par la périphrase FAIRLY IN ACCORDANCE WITH GENERALLY ACCEPTED ACCOUNTING PRINCIPLES ${ }^{38}$.

En revanche, FIDĖLEMENT ne reflète pas la nuance d'impartialité, d'équité de partage qui colore fairly. Mais cette lacune apparente se révèle en fin de compte un avantage, en bloquant l'accès d'une fausse piste. En effet, quelle loi, quel règlement a jamais investi les administrateurs d'une mission d'arbitre? Les états financiers doivent tout au plus viser à rejoindre, au-delà de leurs destinataires immédiats, cet insaisissable « lecteur moyen » dont l'indétermination même répugne à l'idée de partage.

\section{Bien}

L'adverbe BIEN peut, selon le Petit Robert signifier d'une manière satisfaisante, d'une manière conforme à la raison, à la justice, à la morale ou servir d'indicateur de degré ou de quantité. L'adverbe BIEN s'emploie tant pour décrire ce qui est à la limite du correct («Elle danse bien ») que ce qui est admirable ("Un roman bien écrit»). Il joue un rôle emphatique auprès des verbes («Il est bien arrivé à midi »). De plus, il exprime la notion de ce qui est juste et raisonnable : «Agir, se conduire bien. »

Cet adverbe est souple et malléable et, en cela, il conviendrait à l'usage qu'on veut en faire. Il manque par contre d'intensité et il arrive très souvent qu'on sente le besoin de le renforcer pour lui donner plus de signification ( assez bien », «très bien »). Son imprécision et sa faiblesse en font un équivalent peu satisfaisant de fairly.

38. A noter que, selon les renseignements fournis par le Service de Recherche Datum de Soquij (Société québécoise d'information juridique), aucun tribunal, de 1945 à 1973 inclusivement, n'a été amené à faire une interprétation comparative de fairly et de fidèlement. 


\section{Convenablement}

Selon le Petit Robert, l'adverbe CONVENABLEMENT a trois acceptions : l'une vieillie, qui nous renvoie à «opportunément», l'autre familière, qui le définit comme "d'une manière acceptable » par exemple dans «il est payé convenablement $\gg$ et une dernière, sans marque de niveau de langue, qui le rend synonyme de correctement: "Un homme pauvre, mais convenablement vêtu. » Si nous allons voir à l'entrée «convenable», nous remarquons que le premier sens de cet adjectif est littéraire "qui convient, est approprié 》 et on nous renvoie aux adjectifs suivants : adéquat, ad hoc, conforme, congru, convenant, expédiant, idoine, pertinent, propre, à propos. Pour cette acception, on donne en exemple « un parti convenable». Le deuxième sens est familier: "suffisant, acceptable » comme dans "un salaire convenable», "à peine convenable». Enfin, on mentionne que l'acception la plus courante du terme est la suivante « conforme aux règles, aux conventions de la bienséance » et l'on renvoie à * correct, décent, digne, honnête, honorable ». On donne en exemple «des manières convenables», « une tenue, une mise convenable».

Malgré ses emplois littéraire et familier ( qui convient »; «il est payé convenablement $\gg$ ), on ne saurait rejeter ce terme du fait qu'il exprime, tout comme fairly, l'idée de conformité («conforme aux règles »).

\section{CONCLUSION}

Le milieu francophone canadien s'est interrogé plus d'une fois par le passé sur le bien-fondé de l'utilisation du mot fidelement. L'étude qui précède apporte sans doute des éléments propres à expliquer ce phénomène. Elle montre surtout que l'on aurait pu tout aussi bien retenir les adverbes correctement et convenablement que fidèlement. Nos devanciers ont fait un choix. Ils ont opté pour fidelement. En raison de l'importance du rapport du vérificateur et de sa portée juridique, on ne voit pas très bien pourquoi on abandonnerait présentement le mot fidèlement pour un autre. Nous l'avons signalé précédemment, ce mot a, dans notre milieu, une avance sur les autres en raison de son enracinement profond dans l'usage. Comment, en outre, les tribunaux interpréteraient-ils un tel changement?

Nous sommes ainsi conduits à recommander que l'on continue d'employer le mot fidèlement dans le rapport du vérificateur.

Devant un tel aboutissement, on pourrait être tenté de conclure à l'inutilité de notre travail. Nous inclinons, au contraire, à penser qu'il en est autrement. Nous avons cherché à cerner la notion de PRESENT FAIRLY en nous attachant aux éléments centraux qu'ont à l'esprit nos confrères anglophones en utilisant cette expression. Nous avons effectué cette étude avec le souci d'établir une démarche de principe propre à servir éventuellement de guide en d'autres circonstances.

On pourrait en effet se demander quelle devrait être à l'avenir l'attitude des francophones si l'on abandonnait PRESENT FAIRLY, cette expression 
ayant, dans les milieux comptables, une force expressive qui ne s'explique que par la volonté de ces mêmes milieux de lui conférer une telle charge sémantique. Pareille attitude ne repose sur aucune garantie de permanence. Si l'on troquait PRESENT FAIRLY pour une expression qui n'aurait comme objectif que de rendre la même idée que celle qu'on lui attribue présentement, on ne voit pas très bien pourquoi le milieu francophone remplacerait l'adverbe fidèlement. $\mathrm{Si}$, en revanche, l'abandon de PRESENT FAIRLY découlait de la décision de modifier le sens présent et la portée actuelle de l'expression, le milieu francophone serait justifié, à son tour, d'envisager un changement. Un motif de changement pourrait, en outre, venir de l'évolution de nos lois. Si celles-ci tendaient à se rapprocher, par exemple, du modèle français - comme on peut en observer une manifestation dans les règlements de la nouvelle loi fédérale des compagnies - nous croyons que notre milieu pourrait avec pertinence retenir le mot régularité, nous rappelant que «le bilan est régulier lorsqu'il a été établi conformément aux règles de forme et aux méthodes d'évaluation prévues par les textes en vigueur (droit comptable, droit fiscal, droit des sociétés) ${ }^{30} \$$.

Toutefois, nous n'en sommes pas encore là, malgré l'évolution accélérée de ces questions présentement. Si le milieu francophone devait, le cas échéant, s'interroger sur les perspectives que nous venons d'indiquer, nous osons espérer que le présent travail pourrait alors de nouveau être de quelque utilité.

COMITÉ DE TERMINOLOGIE FRANÇAISE DE L'ORdRe DES COMPTABLES AGRÉÉS DU QUÉBEC *

\section{COMPOSITION DU COMITÉ}

Yves-Aubert Côté, C.A., président, Murielle Arsenault, C.A., Louise de Martigny, C.A., André Desrochers, C.A., Claude Dolan, C.A., Robert Dubuc, J.-Paul Michel, C.A., Jean Zalloni, C.A., Carole Gauthier, recherchiste, JeanFrançois Joly, secrétaire de rédaction.

\section{BIBLIOGRAPHIE}

\section{Dictionnaires}

KOHLER, Eric L., A Dictionary for Accountants, Fifth Edition, Englewood Cliff, PrenticeHall, Inc., $1975,498 \mathrm{p}$.

Larousse du $X X^{e}$ siècle, Paris, Librairie Larousse, 1930, 6 volumes.

Petit Robert, Paris, Dictionnaire LE ROBERT, 1970, $1953 \mathrm{p}$.

ROBERT, Paul, Dictionnaire alphabétique et analogique de la langue française, Paris, Société du Nouveau Littré, 1960, 6 tomes.

Livres

GORE, François et Claude DUPOUY, Comptabilité générale de lentreprise industrielle et commerciale, Editions Montchrestien, Paris, 1975 , 929 p.

MAS, Antoine, Introduction à la révision des entreprises, Dunod, Paris, 1959, 351 p.

MEIGS, Walter B., E. John LARSEN et Robert S. MEIGS, Principles of Auditing, Homewood, Richard D. Irwin, Inc., 1973, 777 p.

39. François Goré, Claude Dupouy, Comptabilité générale de l'entreprise industrielle et commerciale, p. 752.

* Le présent rapport n'engage pas la responsabilité de l'Ordre des comptables agrés du Québec. 
MURPHY, Mary E., Auditing and Theory, Homewood, Richard D. Irwin, Inc., 1963, 530 p. SAVATIER, René, le Droit comptable au service de l'homme, Paris, Librairie Dalloz, 1969, $528 \mathrm{p}$.

SMAILS, R.G.H.; Auditing, Fifth Edition, Toronto, Pitman and Sons (Canada) Ltd., 1967, $556 \mathrm{p}$.

STETTLER, Howard S., Auditing Principles, Third Edition. Englewood Cliffs, Prentice-Hall, Inc., $1970,716 \mathrm{p}$.

Périodiques

ECKEL, Leonard G., * The Two-Part Audit Opinion », dans CA magazine, juin 1973.

GORE, François, "Les notions de régularité et de sincérité des comptes », dans Revue française de comptabilité, $\mathrm{n}^{\circ} 25$, avril 1973 , p. 168.

PLEVEN, M., dans la Revue française de comptabilité, octobre 1970, p. 5 et suivantes, note, p. 8.

La Presse de Tunis, 18 septembre 1973.

Monographies

ACCOUNTANTS INTERNATIONAL STUDY GROUP, International Financial Reporting, Brooklyn, Newport Press Inc., 1975.

AUDITING STANDARDS EXECUTIVE COMMITTEE. Statement on Auditing Standards No. 5 - The Meaning of "Present Fairly in Conformity with Generally Accepted Accounting Principles》 in the Independant Auditor's Report, New York, AICPA, 1975, $5 \mathrm{p}$.

$A P B$ Accounting Principles, Original Pronouncements, Vol. 2, Chicago, Commerce Clearing House, Inc., 1973.

COMITE DE RECHERCHE EN COMPTABILITE ET VERIFICATION, Bulletin $n^{\circ} 20$, Toronto, Institut canadien des comptables agrés.

DELL, Bulletin $n^{\circ} 25$, Toronto, Institut canadien des comptables agrees. The Canadian Institute of Chartered Accountants, $1961,143 \mathrm{p}$.

THE GENERAL EDUCATIONAL TRUST OF THE INSTITUTE OF CHARTERED ACCOUNTANTS IN ENGLAND AND WALES, Survey of Published Accounts, 1971-1972, London. The Trustees of Chartered Accountants' Trust for Education and Research of The Institute of Chartered Accountants in England and Wales, 1972, $199 \mathrm{p}$.

ORDIRE DES EXPERTS COMPTABLES ET DES COMPTABLES AGREES, l'Expert comptable dans l'économie nationale et l'Europe de demain, Travaux préparatoires au XXVII Congrès national de l'Ordre des experts comptables et des comptables agrés, Paris, août 1973, $388 \mathrm{p}$.

PUYRAVEAU, M. et M. DESCOTTES-GENON, Incidences du droit fiscal sur la régularité et la sincérité des bilans, Rapport présenté au Congrès de l'Ordre des Conseils fiscaux de France, le 13 mai 1971.

Consultation

M. Pierre-Paul Bourdage, consultant au Service de Recherche Datum de Soquij (Société québécoise d'information juridique), le 8 septembre 1976 . 